\title{
A Systematic Review and Analysis on Deep Learning Techniques Used in Diagnosis of Various Categories of Lung Diseases
}

\author{
Sreedevi Jasthy ${ }^{1}$, Radhakrishna Vangipuram ${ }^{2, \mathbb{Z}}$, Sushama Rani Dutta ${ }^{3}$ \\ ${ }^{1}$ Research Scholar, Department of Computer Science and Engineering, KL University, India \\ ${ }^{2}$ Department of Information Technology, VNR Vignana Jyothi Institute of Engineering and Technology, Hyderabad, India \\ ${ }^{3}$ Assistant Professor, Department of Computer Science and Engineering, KL University, India \\ jsreedevi_cse@klh.edu.in, radhakrishna_v@vnrvjiet.in ${ }^{\not 2}$, sushamadutta@klh.edu.in
}

\begin{abstract}
One of the record killers in the world is lung disease. Lung disease denotes to many disorders affecting the lungs. These diseases can be identified through Chest X-Ray, Computed Tomography CT, Ultrasound tests. This study provides a systematic review on different types of Deep Learning $(D L)$ designs, methods, techniques used by different researchers in diagnosing COVID-19, Pneumonia, Tuberculosis, Lung tumor, etc. In the present research study, a systematic review and analysis is carried by following PRISMA research methodology. For this study, more than 900 research articles are considered from various indexing sources such as Scopus and Web of Science. After several selection steps, finally a 40 quality research articles are included for detailed analysis. From this study, it is observed that majority of the research articles focused on DL techniques with Chest X-Ray images and few articles focused on CT scan images and very few have focused on Ultrasound images to identify the lung disease.
\end{abstract}

Keywords: Lung Diseases, Deep Learning, Prediction, X-Ray, CT, Ultrasound.
Received: 02 November 2021 Accepted: 20 December 2021 Published: 21 December 2021

\section{Introduction}

Lung disease is one of the record killers in the world. Lung disease denotes to many disorders affecting the lungs. Many people around the world are suffering from lung diseases. Common cause of lung disease is smoking, some infections, genes etc. Different types of lung diseases affect different parts of lungs. Airways get affected due to asthma, chronic bronchitis, Chronic Obstructive pulmonary disease (COPD), Emphysema, cystic fibrosis, Acute bronchitis. Air sacs get affected due to Pneumonia, Tuberculosis, Emphysema, Pulmonary edema, Lung cancer, acute respiratory distress syndrome (ARDS), Pneumoconiosis. Interstitium gets affected due to Interstitial lung disease (ILD). Blood vessels get affected due to Pneumonia embolism (PE), pulmonary hypertension. Pleura gets affected due to pleural effusion, pneumothorax, Mesothelioma. Chest wall gets affected due to Obesity hypoventilation, Neuromuscular disorders.

COVID-19 is a new virus which is affecting respiratory system. Scientists believe COVID-19 has similar receptor as severe acute respiratory syndrome (SARS). The first effect of corona virus can be on lungs or on small intestine. Later, it may affect other organs like heart, kidneys, liver, and brain. Lung Ultrasound, CT scan as well as Chest X Ray are the regularly used tests to detect lung diseases. It is a highly challenging task for radiologists and doctors to identify the lung disease. Lung diseases are increasing day by day and there are similarities between different types of lung
Table 1: Top 10 countries with high death rates.

\begin{tabular}{llr}
\hline Rank & Country & Rate \\
\hline 1 & North Korea & 112.26 \\
2 & India & 89.36 \\
3 & Nepal & 77.08 \\
4 & Lesotho & 66.40 \\
5 & Myanmar & 64.49 \\
6 & Bhutan & 63.66 \\
7 & Bangladesh & 61.82 \\
8 & Sao Tome & 60.23 \\
9 & Mali & 54.82 \\
10 & Pakistan & 54.47 \\
\hline
\end{tabular}

diseases, example includes different types of Pneumonia and COVID. Considering such challenges, it becomes difficult for a radiologist or a doctor to accurately identify the disease [38, 39].

This raises a demand for computer aided diagnosis to support the health care system in identifying the disease accurately at an early stage. The Deep Learning (DL) techniques play a key role in carrying this task. The systematic review presented in this paper discusses about different types of deep learning techniques used by various researchers in diagnosing different lung diseases. The statistics of different lung diseases are included in Table 1 which shows the top 10 countries with higher death rates due to lung diseases.

According to WHO, India ranks 2 for deaths due to lung diseases. Categorizing this further, the 


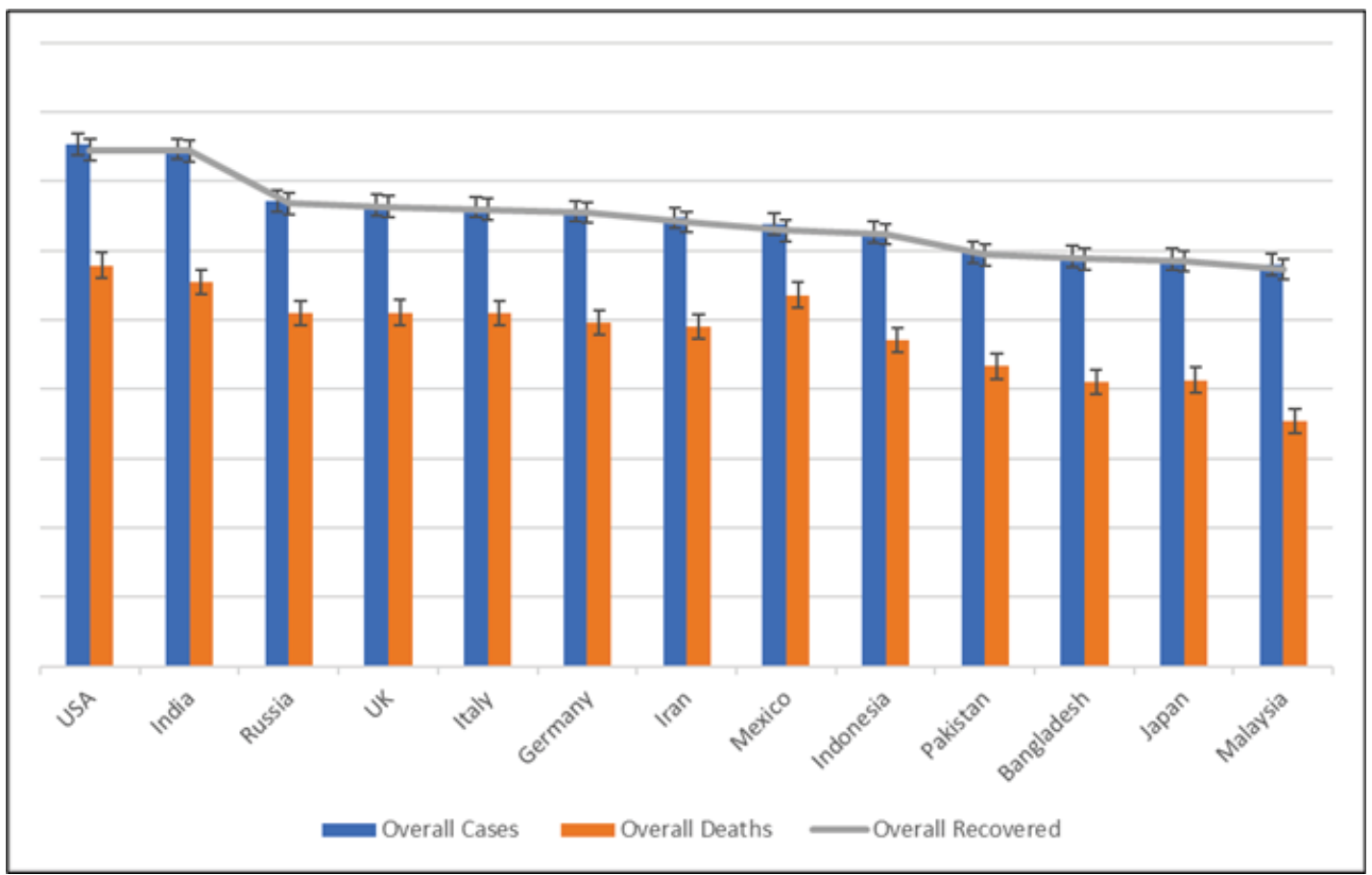

Figure 1: Total covid-19 cases, deaths, and recoveries across 16 countries.

Table 2: Statistics of COVID-19 cases in 16 countries as on 7 th June 2021.

\begin{tabular}{clrrr}
\hline S. & Country & $\begin{array}{r}\text { Overall } \\
\text { Cases }\end{array}$ & $\begin{array}{r}\text { Overall } \\
\text { Deaths }\end{array}$ & $\begin{array}{r}\text { Overall } \\
\text { Recovered }\end{array}$ \\
\hline 1 & USA & $34,211,228$ & 612,378 & $28,122,741$ \\
2 & India & $28,974,152$ & 350,631 & $27,282,022$ \\
3 & Russia & $5,135,866$ & 124,117 & $4,743,202$ \\
4 & UK & $4,522,476$ & 127,841 & $4,277,098$ \\
5 & Italy & $4,233,698$ & 126,588 & $3,918,657$ \\
6 & Germany & $3,709,268$ & 89,872 & $3,542,700$ \\
7 & Iran & $2,971,270$ & 81,183 & $2,565,972$ \\
8 & Mexico & $2,433,681$ & 228,804 & $1,939,596$ \\
9 & Indonesia & $1,863,031$ & 51,803 & $1,711,565$ \\
10 & Pakistan & 933,630 & 21,323 & 864,931 \\
11 & Bangladesh & 812,960 & 12,869 & 753,240 \\
12 & Japan & 762,401 & 13,574 & 706,644 \\
13 & Malaysia & 622,086 & 3,460 & 534,357 \\
14 & Nepal & 591,494 & 7,990 & 497,960 \\
15 & UAE & 585,039 & 1,702 & 564,509 \\
16 & Saudi Arabia & 458,707 & 7,471 & 441,860 \\
\hline & & & &
\end{tabular}

deaths due to Asthma is $1.85 \%$ and India ranks 18; the deaths due to Lung cancers is $1.10 \%$ and India ranks 111; the deaths due to tuberculosis is $4.78 \%$ and India rank 42. These statistics were taken from LUNG DISEASE DEATH RATE BY COUNTRY (worldlifeexpectancy.com) web site. Coronavirus cases recorded so far around the world are 174,093,954, which include 3,745,104 deaths and $57,124,854$ recoveries. Table 2 shows the statistics of Corona virus in 16 countries as on 7th June 2021. (www.worldmeters.info/coronavirus). Fig. 1 depicts the same data in graph.

The diagnosis of COVID -19 through RT-PCR test takes nearly $28 \mathrm{hrs}$, CT scan takes nearly 30 minutes and CXR takes nearly 5 minutes. Though CXR gives faster results, the diagnosis may go wrong due to much similarity between CXRs of Pneumonia and COVID19. Thus similarity function plays crucial role in learning models [2]. Considering all these, this paper focuses on different ML and DL techniques used in classifying the diseases accurately and early.

The following questions are considered in this study:

- Can DL assist radiologists/doctors in diagnosing the lung disease?

- What are different DL algorithms commonly used for lung disease detection?

- What are different types of lung images that can be used to train the DL models?

This paper consists of three sections. First section discusses on Research Methodology. Second section discuss different algorithms used in the study and as well as different datasets and number of classes considered in classifying the disease. Third section consolidates different metrics used to evaluate the algorithms. Last section provides conclusion based on this study.

\section{Research Methodology}

The research methodology that is carried out for performing systematic review is reported using PRISMA flow diagram. It depicts all the steps followed from identification of the articles to the selection of eligible articles for the survey. The PRISMA flow diagram is shown in Fig. 2. 


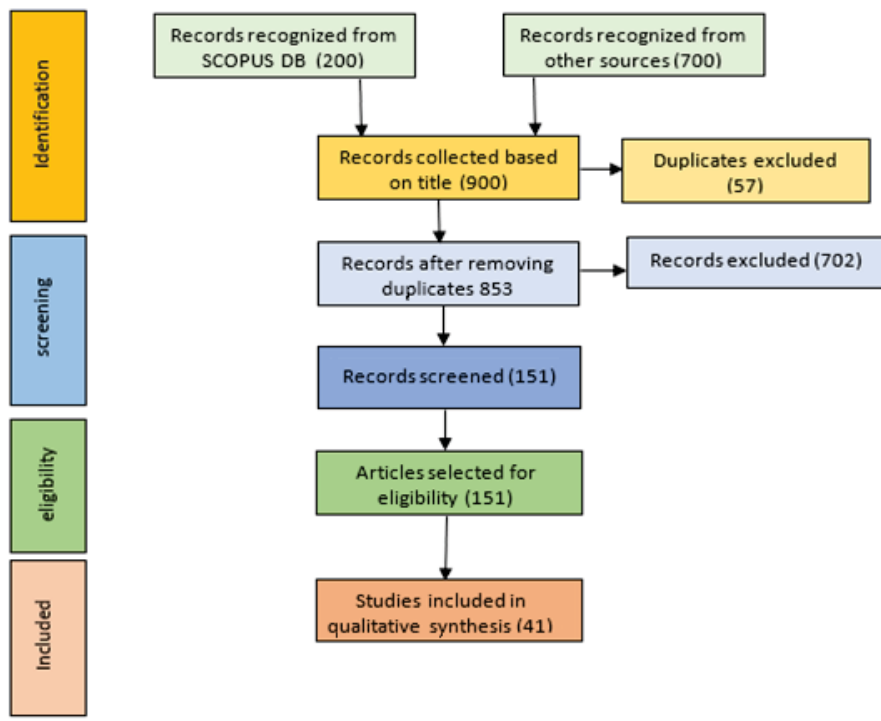

Figure 2: PRISMA (Preferred Reporting Items for Systematic Reviews and Meta-Analyses) for selecting the articles (http://www.prisma-statement.org/).

Table 3: Number of articles chosen from 2014-2021.

\begin{tabular}{cc}
\hline Year & No. of Articles Chosen \\
\hline 2021 & 3 \\
2020 & 24 \\
2019 & 6 \\
2018 & 2 \\
2017 & 5 \\
2015 & 1 \\
\hline
\end{tabular}

\section{Article Sources}

Research articles are downloaded from different indexing sources like Scopus database and other sources as listed in the Table 4. A total of 900 articles are recognized initially. From these 900 articles, duplicate articles from different sources and those which are not relevant are excluded from consideration.

In next step, articles which have high citations were only considered. Further, articles having high citations but not certified by peer review were excluded. As accurate detection of lung disease is the focus, the articles which used CXR, CT, Ultrasound images are considered in this study. These are chosen so that the reliability of this systematic review is not compromised.

\section{Data Collection Process}

The articles from 2014 to 2021 are considered in this survey as shown in Table 4 . The articles which are having high citations were considered. The articles which have high citations but not yet published are not considered. The key words used in this survey include:

- Lung disease classification using DL

- Lung disease diagnosis using DL
Table 4: Source of articles.

\begin{tabular}{lc}
\hline Publisher & No. of Articles \\
\hline ELSEVIER & 17 \\
ersJournals & 1 \\
Hindawi & 2 \\
IEEE Access & 5 \\
MDPI & 1 \\
Nature & 4 \\
NCBI & 1 \\
RSNA & 4 \\
Springer & 5 \\
\hline
\end{tabular}

- Lung disease identification using DL

- Lung disease identification using DL with metrics

\section{DL Techniques Used in This Study}

\section{Introduction to Deep Learning}

Advances in image acquisition devices over past few years, to provide high resolution images, have improved health care system by allowing doctors to diagnose the diseases more efficiently. But as the amount of data is quite large, image analysis becomes more and more tedious and complex job for radiologists and doctors. Additionally, results can be human error prone and inconsistent. Hence, extensive research is happening in this field to automate the medical image analysis and diagnosis. AI can learn from huge set of medical images and is estimated to be $10 \%$ more accurate than an average radiologist [38, 39].

Conventional ML techniques based on supervised learning are not suitable to analyze this complex, massive and varying data. When compared to other ML 


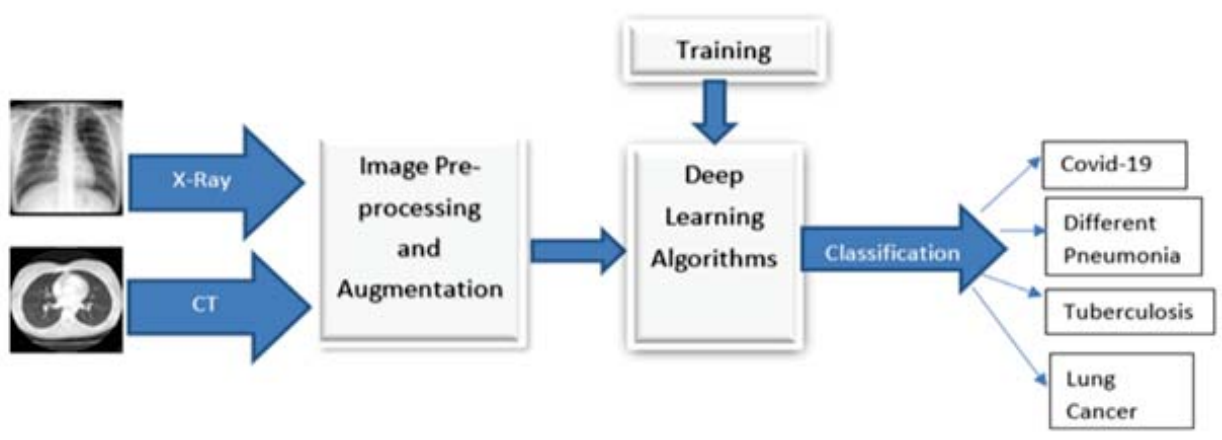

Figure 3: Block diagram of classification of lung diseases using DL.

techniques, DL is considered to have an added benefit of being able to take decisions with very less involvement from human trainers. DL is an ML technique which is based on structure of human brain. In recent times DL is getting more attention in different fields including medical imaging. DL uses layered architecture with one input, multiple hidden, and one output layers and is based on artificial neural networks. It automatically learns from massive amounts of data and as it continues to processes more and more data, it becomes more and more accurate. In medical imaging, DL can be used for various purposes like classification, image segmentation, object detection, data augmentation of limited data sets, and image transformation. Some of the DL frameworks include Microsoft cognitive toolkit, Pytorch, DLJ4, Caffe, TensorFlow, Keras.

\section{General Flow of DL Algorithms}

A general flow of DL for imaging is depicted in Fig. 3. The first task is to acquire images. Once the image is acquired using the imaging techniques, it is processed through a computer aided analysis to obtain proper medical diagnosis.

The initial step in any image processing flow is the Pre-processing to reduce/remove the noise, blur, unwanted features from the image. Frequently used preprocessing techniques are:

Filtering. Filtering aims at removing unwanted noise, blur, features to get better visualization of actual region of interest.

Segmentation. The objective of segmentation is to divide the image into regions with similar features.

Data Augmentation. Training of neural networks require massive amounts of data which is difficult to obtain due to many reasons. So, Data augmentation techniques, which apply various transformations (by adding noise, rotations, contrast, etc.), to produce different variations of datasets from available limited data.

Once the image is pre-processed, it is passed through DL algorithm to classify it into predefined labels. The DL algorithm is pre-trained and continues to learn while it is being used for classification of new images

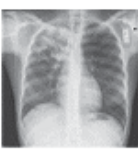

a)

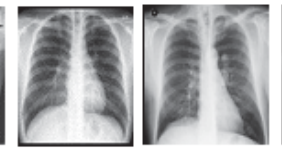

b) c)

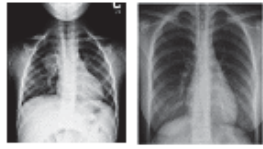

d) e)
Figure 4: Sample X-ray images (a) Normal Chest XRay (b) Tuberculosis Lung X- Ray (c) Lung Cancer XRay (d) Pneumonia X-Ray images were collected from Radiopaedia.org (e) COVID-19 X-Ray image [7].

[23].

In DL, transfer learning is an important technique where the neural network is trained on a task where there is abundant data, and then the weights from this network are copied to network of actual task. This helps to obtain good accuracy even with limited data.

The advances in AI, has made it possible to apply the medical imaging models not only to latest digital images like CT or MRI but also to conventional images like X-rays and endoscopy images. The study focuses on lung disease diagnosis which predominantly uses CT / X-ray imaging. The X-ray and CT images shown in Fig. 4 and 5 are a sample of how normal and diseaseaffected images look like.

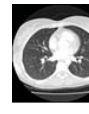

a)

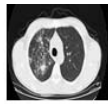

b)

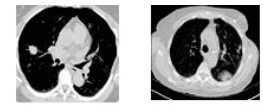

d)

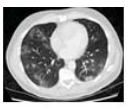

e)
Figure 5: CT scan images of different lung diseases (a) Normal (Radiopaedia.org) (b) Tuberculosis (Radiopaedia.org) (c) Lung Cancer (Science Photo Library) (d) Eosinophilic pneumonia (Science Photo Library) (e) Coronavirus (radiogyan.com).

\section{Neural Networks}

Neural networks are inspired from human neural system and use artificial intelligence for deep learning. They consist of multiple layers of interconnected neurons that are powered by activation function [6]. At high level it is composed of 3 layers: one input, multiple hidden and one output layers. Hidden layers take 
Table 5: Acronyms.

\begin{tabular}{ll}
\hline DL & Deep Learning \\
CNN & Convolutional Neural Network \\
Reg-STN & Regularised Spatial Transformer Networks \\
SORD & Soft Ordinal regression \\
DBN & Deep Belief Network \\
DCNN & Deep Convolutional Neural Network \\
DECoVNet & $\begin{array}{l}\text { Deep Convolutional Neural Network } \\
\text { to detect COVID 19 }\end{array}$ \\
DenseNet & Densely Connected Networks \\
DeTraC & Decompose Transfer and Compose \\
DFCNet & Deep Flow Collaborative Network \\
DLAD & Deep Learning-based automatic \\
DNN & detection algorithm \\
HSCNN & Deep Neural Network \\
MAN & Nerarchical Semantic Convolutional \\
MC-CNN & Modified AlexNet \\
MODE & Multi-crop Convolutional Neural Network \\
ResNet & Evolti-Objective Differential \\
ODNN & Residual Networks \\
SAE & Optimal Deep Neural Network \\
LIDC-IDRI & Stacked Autoencoder \\
& Consortium image collection \\
\hline
\end{tabular}

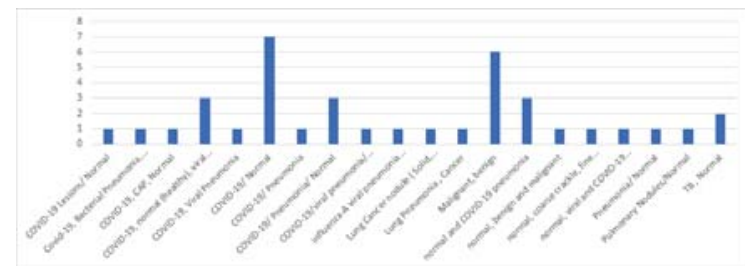

Figure 6: Graph represents different dataset classes used by authors in the study.

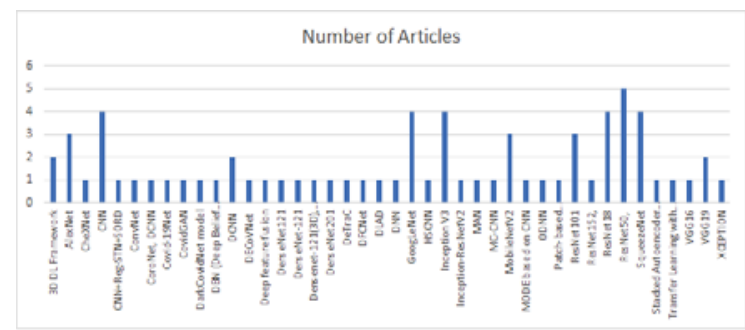

Figure 7: DL algorithms referred in different articles.

the input and produce output using activation function.

There are different types of neural networks based on their structure, depth, types of neurons used. For different types of Neural Networks, see [9]. Table 6 shows the brief review of the articles.

\section{Datasets}

Datasets used in reviewed articles are from public sources like Kaggle, Git Hub, LIDC-IDRI and few are collected from different hospitals. Table 7, which can be found in the supplement shows the details of the dataset. The summary of different classes used by researchers is shown in Table 8 (which can be found in the supplement) and Fig. 7. Article [7] has considered 7 classes, [13] refer to 6 classes, [27, 44] refer to 5 classes, $[26,18,11]$ refer to 4 classes, $[28,37,27,20,21,18]$ refer to 3 classes and $[45,28,34,24,40,31,5,17,15$, 8, 32, 25, 22, 41, 35, 16, 19, 29, 47, 43, 18, 12, 4, 46, 36] refer to 2 classes.

\section{Data Augmentation}

Data Augmentation aims at increasing the size of dataset. In DL, large number of datasets are required for training the model. As publicly available data is very less in clinical research, data augmentation is applied for images to obtain good accuracy. Data augmentation is used in different articles: [45, 15, 27, 8, 32, $22,7,19,41,35,1,21,47,33,12,13,46,11,40,36,29]$.

\section{Techniques Referred in the Articles}

Table 9 and Fig. 7 shows different DL techniques used in different articles.

\section{AUC-ROC Curve}

The Receiver Operator Characteristic (ROC) curve is a metric that assesses the ability of the model to distinguish between binary classes.

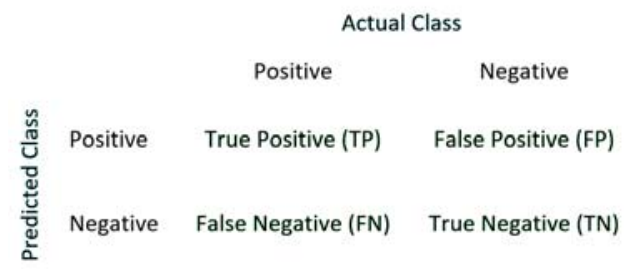

Figure 8: Confusion Matrix.
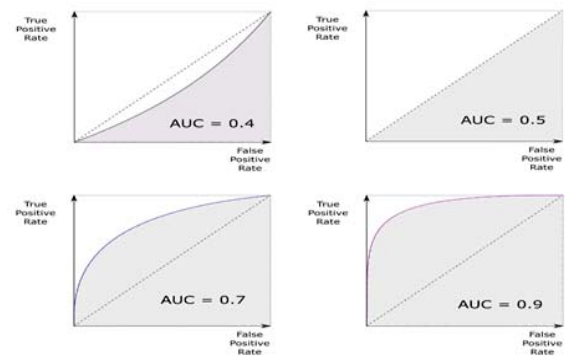

Figure 9: Represents sample Area Under Curve. 
Table 6: Brief review of different articles.

\section{Ref Remarks}

Lung area is identified and sent to COVID-19 Net for diagnosing and predicting Covid-19 disease from different forms of pneumonia. But on deeper analysis, the features have become abstract in this model In this method 3D CT volumes are used. This approach follows a 2-step process. First step used UNet which was already trained, for separation of

[45] lung region. In second step this segmented image was passed to a "3D based Deep Neural Network" to predict covid-19 disease. The drawback was not using CT images of pneumonia patients which are in general difficult to segregate from those of covid-19 affected patients.

15] Lung region was identified from CT images. 3D and Hybrid 3D models are applied to classify Covid-19 or Pneumonia. High accuracy is achieved but model training is limited to covid -19 and pneumonia and there is a moderate decrease in sensitivity

T28 The model 'DarkCovidNet' is based on DarNet but uses reduced number of filters and layers. The results obtained are promising for good quality X-rays but not up to mark for those with low-quality.

34] The model used is MODE-based CNN with 20-fold cross-validation. Main intention of using cross-validation is to avoid overfitting. When test

results of proposed model are compared against those of other competitive models, it was proved that this model yields better results. This method helps to identify chest images as - covid-19 affected or pneumonia affected or normal. This approach is composed of multiple steps, first being the elimination of noise through Fuzzy Colour technique. Second step aims at developing an image with improved quality, which is achieved by

37] 'Stacking' (combining) the recreated images in first step with the original image. Then the stacked image is passed through couple of deep learning models - "MobileNetV2" and "SqueezeNet". Later the thousand features extracted by deep learning models are reduced to few significant features by using SMO algorithm. Finally SVM is used for classification. The approach involved multiple phases to develop a large database. 'Feature extraction' is the initial step which is followed by 'Early Fusion',

[30] 'Resampling' and finally 'Classification'. The idea is that a large database yield better results when passed through Deep learning algorithms with appropriate depth.

27] This paper proposes patch-level classification using deep neural network for this purpose. Once the patch-level classes are obtained, they are combined using majority voting to obtain the final class

31] Spatial Transformer Networks are used to estimate the severity of disease and to identify the region of disease.

In the proposed approach DL techniques are used - 1) for classification and 2) for combining learned and hand-crafted features in order to achieve

[8] better classification accuracy. The DL method used for classification is known as 'Modified AlexNet' (MAN) and is based on SVM. Its performance is compared against 'SoftMax', 'ResNet50', 'AlexNet', 'VGG16' and 'VGG19'.

The proposed approach 'HSCNN' is a 2-level prediction model which improves the overall prediction accuracy and provides better explanation of diagnosis. At first level it classifies the nodules based on their semantic characteristics. The features from semantic feature prediction are fed to second level which predicts the final malignancy. In addition to improved accuracy the advantage of this model is that the first level can be used independently as a semantic feature generator and can be unified with other malignancy prediction models

This method is a CNN model with depth of 25 layers and 8 residual connections. First segmentation accurately identifies the lung region. Then the

25] proposed algorithm 'DLAD' is trained with same dataset but 3 different hyper parameters. The mean of results from these 3 networks gives the final class. The advantage of DLAD is accurate detection of malignant nodules, while the disadvantage is, it is not optimized to distinguish between benign and non-benign nodules.

In this model initially ODNN and LDA are applied to extract the features from CT image. Then LDR is used for classification. The model gives sensitivity, specificity and accuracy of $96.2 \%, 94.2 \%$ and $94.56 \%$ respectively.

In this approach 'DFCNet' is used for classification at two levels. In first level, the algorithm classifies the CT image as either cancerous or normal.

[22] At second level the cancerous image is further classified into 4 stages of lung cancer. Usage of data augmentation further improved the training process of DFCNet.

This is a special approach which uses lung sounds to classify the underlying disorders. The dataset has 7 classes of sounds which map to different

7] lung disorders. This paper compared the performance for three ML approaches. Two of these approaches are based on classification algorithms like SVM, KNN and Gaussian mixture models (GMM) while the last one is CNN based. The results indicate that CNN approach is better. This approach is based on Deep CNNs. It uses 'AlexNet' and 'GoogleNet' to categorize the given lung image as either ordinary or TB-affected. The

[19] images from ImageNet were used for training and testing. Multiple pre-processing techniques are applied to obtain image augmentation. Once best performing algorithms are identified, these are combined (ensemble) to achieve better predictive performance. The proposed approach includes multiple steps. Initial step is suppression of unwanted portions of image like ribs using 'PCA' (Principal Component

41] Analysis) for better visibility of lungs. The next step is image segmentation using 'Active Shape Model'. The next step uses 'Laplacian of Gaussian' to derive candidate nodules. Both hand-crafted and DL based methods are used to obtain features of candidate nodules. The obtained features are used to train the 'Cost Sensitive Random Forest' classifier is used for classification.

The paper evaluates 3 neural networks - CNN, DNN, SAE - in classification of input CT images as benign or malignant. The obtained results indicate that CNN is the best among the three algorithms with accuracy, sensitivity of and specificity of $84.5 \%, 83.96 \%$ and $84.32 \%$ respectively.

[16] Owing to the advantage of automatic feature exploitation and performance tuning in CNN and deep belief network models over the conventional methods, this paper presents a simplified image analysis and classification pipeline for CT images based on CNN.

In this paper, five CNN based models are evaluated with three types of binary datasets and 'ResNet50' proved to achieve high accuracy. First dataset

[26] includes data for covid-19 and normal; second dataset includes data for covid-19 and viral pneumonia; third dataset includes data for covid-19 and bacterial pneumonia

[1] DeTraC model is used to detect covid-19. DeTrac has an advantage of handling irregularities in the dataset by using a class decomposition mechanism.

ResNet18, ResNet50, SqueezeNet, and DenseNet-121 algorithms were used on 5000 chest images - 2000 for training and 3000 for testing. About

$98 \%$ accuracy was achieved.

[21] A 3D deep learning framework was developed to diagnose covid-19 disease. Accuracy is high but the article focuses on CAP and Covid -19. Other pneumonia cases were not considered.

47] CT images were taken as input and pre-processing is done based on Hounsfield units. Candidate region segmentation is done by using 3DCNN and Image classification model is used for finding the candidate region.

[13] CT images were collected from different hospitals and in the first step pre-processing is done, next feature extraction and finally classification is done to find covid-19 or viral pneumonia. Training dataset considered is very small in this study.

[33] MC_CNN model is used which learns the deep features of image to detect malignancy and diameter of affected region. In the proposed method a chest X-ray image is passed through CNN based algorithm 'CoroNet' to identify covid-19 infection. Though the proposed

[18] algorithm is less expensive and delivered promising results when tested with limited dataset, it is expected to be tested with large dataset to confirm its performance.

12] Shallow networks and deep networks algorithms were evaluated in this article, and it was observed that DenseNet201 outperforms well than the remaining algorithms

[13] Automatic nodule classification is done. For this ConvNets framework is designed and it is compared with SVM and K-Means algorithm. The results were observed by two radiologists who have more than 20 years of experience.

[46] A 2D CNN model is proposed to identify the pulmonary nodules. Here 3 models are trained in order to obtain accurate model.

[4] This paper explores usage of CNN based DL for detection and extraction of features for covid-19 disease from X-ray images.

[11] Pre-processing, Data augmentation and Transfer learning are used in order to classify the image into predefined labels.

[40] Proposes a model called CovidGAN which has improved performance than CNN for covid-19 detection.

[36] This paper proposes a 2-lvel approach of feature extraction and then classification. A CNN model are binary indicating if person is affected by pneumonia or not. The input used is X-ray images.

[29] In this paper, author proposed a simple CNN based model. Based on the tests performed, the model gave better performance than earlier models.

[10] This method uses high resolution CT images to predict if the patient is affected by covid-19. The model uses DL based algorithm and gives an accuracy of $95.24 \%$ per patient and an accuracy of $98.85 \%$ per image.

A model named 'Inf-Net; is proposed to detect covid-19 infected regions from CT images. In this method once features are extracted, they

[14] are aggregated to build a global map. To overcome the disadvantage of a smaller number of labelled images, a segmentation algorithm using semi-supervised learning is used, which works well even with unlabeled data.

[5] 10 Well known CNNs are used to differentiate covid-19 and non-covid-19 infections and their performance is evaluated by with well-known techniques.

MENDEL - Soft Computing Journal, Volume 27, No. 2, December 2021, Brno, Czech Republic 


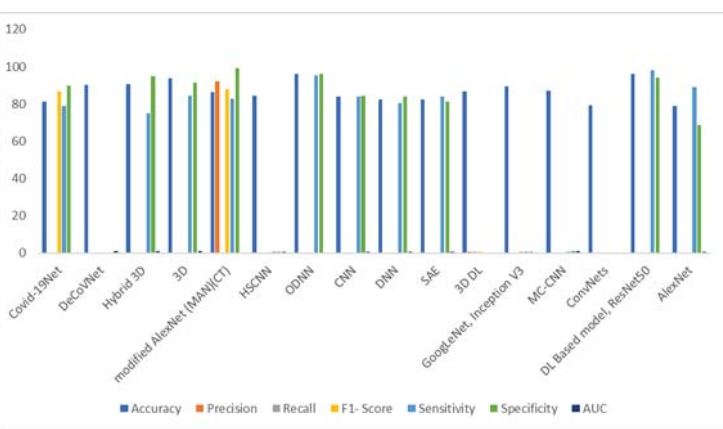

Figure 10: Accuracy, Precision, Recall, F1-Score, Sensitivity, Specificity of different DL when applied for CT images.

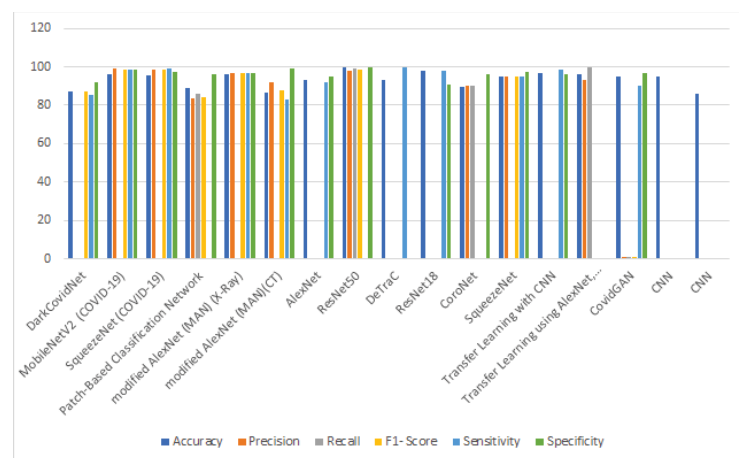

Figure 11: Accuracy, Precision, Recall, F1-Score, Sensitivity, Specificity of different DL when applied for X-Ray Images.

\section{Metrics}

Metrics help in assessing the performance of the model. Table 10 shows the metrics used in assessing the performance of the learning model.

Confusion matrix displays number of correctly and in-correctly predicted classes. The size of confusion matrix is based on classes. If the classes to be predicted is ' $\mathrm{N}$ ', then size of confusion matrix is ' $\mathrm{NxN}$ '. The confusion matrix gives simple way to compare the correctly predicted classes vs incorrectly predicted classes and thereby helps to get a fair idea of accuracy of the algorithm.

Once the values for TP, NP, FP and FN are known, we can derive metrics essential for assessment of classification model as shown in the below Table 10. The plot of ROC curve is shown in Fig. 9. This curve is created by plotting the True Positive Rate (TPR) against the False Positive Rate (FPR) at various threshold settings.

A classifier with ROC curve above the diagonal is said to be good classifier. The performance of the model improves if it becomes skewed towards the upper left corner. The Area Under the Curve (AUC) is the measure of the area under the ROC curve. Like ROC, AUC also measures the ability of a classifier to distinguish between binary classes. The higher the AUC the better the model is at predicting the binary classes. Ta-
Table 9: DL techniques used in different articles.

\begin{tabular}{|c|c|c|}
\hline Ref. No & Deep Learning Algorithm & $\begin{array}{l}\text { Number of } \\
\text { Articles }\end{array}$ \\
\hline$[21,47]$ & 3D DL Framework & 2 \\
\hline$[19,11,5]$ & AlexNet & 3 \\
\hline$[12]$ & CheXNet & 1 \\
\hline$[35,16,36,29]$ & $\mathrm{CNN}$ & 4 \\
\hline [31] & $\mathrm{CNN}+$ Reg-STN+SORD & 1 \\
\hline$[13]$ & ConvNet & 1 \\
\hline$[18]$ & CoroNet, DCNN & 1 \\
\hline$[44]$ & Covid-19Net & 1 \\
\hline$[40]$ & CovidGAN & 1 \\
\hline$[28]$ & DarkCovidNet model & 1 \\
\hline$[16]$ & DBN (Deep Belief Network) & 1 \\
\hline$[19,12]$ & DCNN & 2 \\
\hline$[45]$ & DECoVNet & 1 \\
\hline [41] & Deep feature fusion & 1 \\
\hline$[11]$ & DenseNet121 & 1 \\
\hline$[24]$ & DenseNet-121 & 1 \\
\hline$[15]$ & Densenet-121(3D), Hybrid 3D & 1 \\
\hline$[12]$ & DenseNet201 & 1 \\
\hline [1] & DeTraC & 1 \\
\hline$[22]$ & DFCNet & 1 \\
\hline$[25]$ & DLAD & 1 \\
\hline$[35]$ & DNN & 1 \\
\hline$[19,43,11,5]$ & GoogLeNet & 4 \\
\hline$[32]$ & HSCNN & 1 \\
\hline$[26,43,12,11]$ & Inception V3 & 4 \\
\hline [26] & Inception-ResNetV2 & 1 \\
\hline$[8]$ & MAN & 1 \\
\hline [33] & $\mathrm{MC}-\mathrm{CNN}$ & 1 \\
\hline$[37,12,5]$ & MobileNetV2 & 3 \\
\hline [34] & MODE based on CNN & 1 \\
\hline$[20]$ & ODNN & 1 \\
\hline$[27]$ & Patch-based CNN & 1 \\
\hline$[26,12,5]$ & ResNet101 & 3 \\
\hline$[26]$ & ResNet152, & 1 \\
\hline$[24,12,48]$ & ResNet18 & 4 \\
\hline$[26,24,21,10]$ & ResNet50, & 5 \\
\hline$[37,24,12,5]$ & SqueezeNet & 4 \\
\hline$[35]$ [3 - & Stacked Autoencoder (SAE) & 1 \\
\hline$[4]$ & Transfer Learning with CNN & 1 \\
\hline$[5]$ & VGG16 & 1 \\
\hline$[12,5]$ & VGG19 & 2 \\
\hline [5] & XCEPTION & 1 \\
\hline
\end{tabular}

Table 10: Metrics in assessing the performance.

\begin{tabular}{|c|c|c|}
\hline Measure & Definition & Formula \\
\hline Accuracy & $\begin{array}{l}\text { Represents the number } \\
\text { of right predictions }\end{array}$ & $\frac{T P+T N}{T P+T N+F P+F N}$ \\
\hline Precision & $\begin{array}{l}\text { Represents positive } \\
\text { cases rightly identified }\end{array}$ & $\frac{T P}{T P+F P}$ \\
\hline Recall or & Proportion of active & \\
\hline Sensitivity & $\begin{array}{l}\text { positive cases that are } \\
\text { rightly identified }\end{array}$ & $\frac{T P}{T P+F N}$ \\
\hline F1-Score & $\begin{array}{l}\text { Geometric mean of pre- } \\
\text { cision and recall }\end{array}$ & $\frac{2 \cdot \text { Precision } \cdot \text { Recall }}{\text { Precision }+ \text { Recall }}$ \\
\hline Specificity & $\begin{array}{l}\text { Amount of actual nega- } \\
\text { tive cases that are pre- } \\
\text { dicted as negative }\end{array}$ & $\frac{T N}{T N+F P}$ \\
\hline
\end{tabular}


ble 11, which can be found in the supplement, gives the metrics of DL algorithms for X-Ray and CT images.

\section{Conclusion}

A systematic search is performed in identifying the articles related to lung diseases. Model ResNet50 [26] for $\mathrm{X}$-Ray images gives highest accuracy of $99.5 \%$. The classes considered in the dataset are COVID-19, normal (healthy), viral pneumonia and bacterial pneumonia. Model ODNN [20] and ResNet50 [10] gives highest accuracy of $96 \%$ for CT images. The classes considered in this [20] are normal, benign and malignant and in [10] the classes are COVID-19, Pneumonia and Normal. However, in perspective of clinical research, models should be evaluated with clear criteria with the help of radiologists, prior to applying them. As dayby-day death rate is increasing because of respiratory related diseases, the ML and DL techniques can help the health care professionals in identifying the disease in early stage.

\section{References}

[1] Abbas, A., Abdelsamea, M. M., And Gaber, M. M. Classification of covid-19 in chest x-ray images using detrac deep convolutional neural network. Applied Intelligence 51, 2 (2021), 854-864.

[2] Aljawarneh, S., Radhakrishna, V., And KuMAR, G. R. An imputation measure for data imputation and disease classification of medical datasets. In AIP Conference Proceedings (2019), vol. 2146, AIP Publishing LLC, p. 020001.

[3] Alquran, H., Alsleti, M., Alsharif, R., Qasmieh, I. A., Alqudah, A. M., And Harun, N. H. B. Employing texture features of chest $\mathrm{x}$ ray images and machine learning in covid-19 detection and classification. MENDEL Journal 2\%, 1 (2021), 9-17.

[4] Apostolopoulos, I. D., and Mpesiana, T. A. Covid-19: automatic detection from x-ray images utilizing transfer learning with convolutional neural networks. Physical and Engineering Sciences in Medicine 43, 2 (2020), 635-640.

[5] Ardakani, A. A., Kanafi, A. R., Acharya, U. R., Khadem, N., and Mohammadi, A. Application of deep learning technique to manage covid-19 in routine clinical practice using ct images: Results of 10 convolutional neural networks. Computers in biology and medicine 121 (2020), 103795.

[6] Bandaru, R., Pola, S., Thadem, S. A., Pendyala, K., VAngipuram, R., And VANGIPURAM, S. K. Design and analysis of activation functions used in deep learning models. In The 7th International Conference on Engineering \& MIS 2021 (2021), pp. 1-5.

[7] Bardou, D., Zhang, K., And Ahmad, S. M. Lung sounds classification using convolutional neural networks. Artificial intelligence in medicine 88 (2018), 58-69.

[8] Bhandary, A., Prabhu, G. A., Rajinikanth, V., Thanaraj, K. P., Satapathy, S. C., Robbins, D. E., Shasky, C., Zhang, Y.-D., Tavares, J. M. R., and RaJa, N. S. M. Deeplearning framework to detect lung abnormality-a study with chest x-ray and lung ct scan images. Pattern Recognition Letters 129 (2020), 271-278.

[9] Bishop, C. M., ET AL. Neural networks for pattern recognition. Oxford university press, 1995.

[10] Chen, J., Wu, L., Zhang, J., Zhang, L., Gong, D., Zhao, Y., Chen, Q., Huang, S., YANG, M., YANG, X., ET AL. Deep learningbased model for detecting 2019 novel coronavirus pneumonia on high-resolution computed tomography. Scientific reports 10, 1 (2020), 1-11.

[11] Chouhan, V., Singh, S. K., Khamparia, A., Gupta, D., Tiwari, P., Moreira, C., Damaševičius, R., And De Albuquerque, V. H. C. A novel transfer learning based approach for pneumonia detection in chest x-ray images. $A p$ plied Sciences 10, 2 (2020), 559.

[12] Chowdhury, M. E., Rahman, T., KhanDakar, A., Mazhar, R., Kadir, M. A., MaHBub, Z. B., Islam, K. R., Khan, M. S., Iqbal, A., Al Emadi, N., ET AL. Can ai help in screening viral and covid-19 pneumonia? IEEE Access 8 (2020), 132665-132676.

[13] Ciompi, F., Chung, K., Van Riel, S. J., Setio, A. A. A., Gerke, P. K., Jacobs, C., Scholten, E. T., Schaefer-Prokop, C., Wille, M. M., Marchiano, A., ET Al. Towards automatic pulmonary nodule management in lung cancer screening with deep learning. Scientific reports 7, 1 (2017), 1-11.

[14] Fan, D.-P., Zhou, T., Ji, G.-P., Zhou, Y., Chen, G., Fu, H., Shen, J., And Shao, L. Infnet: Automatic covid-19 lung infection segmentation from ct images. IEEE Transactions on Medical Imaging 39, 8 (2020), 2626-2637.

[15] Harmon, S. A., SAnford, T. H., Xu, S., Turkbey, E. B., Roth, H., Xu, Z., Yang, D., Myronenko, A., Anderson, V., Amalou, A., ET AL. Artificial intelligence for the detection of covid-19 pneumonia on chest ct using multinational datasets. Nature communications 11, 1 (2020), 1-7.

[16] Hua, K.-L., Hsu, C.-H., Hidayati, S. C., Cheng, W.-H., And Chen, Y.-J. Computeraided classification of lung nodules on computed tomography images via deep learning technique. OncoTargets and therapy 8 (2015).

[17] Huang, L., Han, R., Ai, T., Yu, P., Kang, H., TAO, Q., AND XIA, L. Serial quantitative chest ct assessment of covid-19: a deep learning approach. Radiology: Cardiothoracic Imaging 2, 2 (2020), e200075. 
[18] Khan, A. I., Shah, J. L., And Bhat, M. M. Coronet: A deep neural network for detection and diagnosis of covid-19 from chest x-ray images. Computer Methods and Programs in Biomedicine 196 (2020), 105581.

[19] Lakhani, P., And Sundaram, B. Deep learning at chest radiography: automated classification of pulmonary tuberculosis by using convolutional neural networks. Radiology 284, 2 (2017), 574582.

[20] Lakshmanaprabu, S., Mohanty, S. N., Shankar, K., Arunkumar, N., and Ramirez, G. Optimal deep learning model for classification of lung cancer on ct images. Future Generation Computer Systems 92 (2019), 374-382.

[21] Li, L., Qin, L., Xu, Z., Yin, Y., Wang, X., Kong, B., BAI, J., Lu, Y., FAng, Z., Song, Q., ET AL. Artificial intelligence distinguishes covid-19 from community acquired pneumonia on chest ct. Radiology (2020).

[22] Masood, A., Sheng, B., Li, P., Hou, X., Wei, X., QIn, J., AND Feng, D. Computer-assisted decision support system in pulmonary cancer detection and stage classification on ct images. Journal of biomedical informatics 79 (2018), 117-128.

[23] Matousek, R., Dobrovsky, L., And Kudela, J. How to start a heuristic? utilizing lower bounds for solving the quadratic assignment problem. International Journal of Industrial Engineering Computations 13, 2 (2022), 151-164.

[24] Minaee, S., Kafieh, R., Sonka, M., Yazdani, S., AND Soufi, G. J. Deep-covid: Predicting covid-19 from chest x-ray images using deep transfer learning. Medical image analysis 65 (2020), 101794.

[25] Nam, J. G., Park, S., Hwang, E. J., Lee, J. H., Jin, K.-N., Lim, K. Y., Vu, T. H., Sohn, J. H., Hwang, S., Goo, J. M., et AL. Development and validation of deep learningbased automatic detection algorithm for malignant pulmonary nodules on chest radiographs. $R a-$ diology 290, 1 (2019), 218-228.

[26] Narin, A., Kaya, C., And Pamuk, Z. Automatic detection of coronavirus disease (covid-19) using x-ray images and deep convolutional neural networks. Pattern Analysis and Applications (2021), 1-14.

[27] Oh, Y., Park, S., and Ye, J. C. Deep learning covid-19 features on cxr using limited training data sets. IEEE transactions on medical imaging 39, 8 (2020), 2688-2700.

[28] Ozturk, T., Talo, M., Yildirim, E. A., Baloglu, U. B., Yildirim, O., ANd Acharya, U. R. Automated detection of covid-19 cases using deep neural networks with x-ray images. Computers in biology and medicine 121 (2020), 103792.

[29] Pasa, F., Golkov, V., Pfeiffer, F., CreMERs, D., AND PfEIfFER, D. Efficient deep network architectures for fast chest x-ray tuberculosis screening and visualization. Scientific reports 9, 1 (2019), 1-9.

[30] Pereira, R. M., Bertolini, D., Teixeira, L. O., Silla JR, C. N., and Costa, Y. M. Covid-19 identification in chest $\mathrm{x}$-ray images on flat and hierarchical classification scenarios. Computer Methods and Programs in Biomedicine 194 (2020), 105532.

[31] Roy, S., Menapace, W., Oei, S., Luijten, B., Fini, E., Saltori, C., Huijben, I., ChennakeShava, N., Mento, F., Sentelli, A., et Al. Deep learning for classification and localization of covid-19 markers in point-of-care lung ultrasound. IEEE transactions on medical imaging 39, 8 (2020), 2676-2687.

[32] Shen, S., Han, S. X., Aberle, D. R., Bui, A. A., And Hsu, W. An interpretable deep hierarchical semantic convolutional neural network for lung nodule malignancy classification. Expert systems with applications 128 (2019), 84-95.

[33] Shen, W., Zhou, M., Yang, F., Yu, D., Dong, D., YAng, C., ZAng, Y., And Tian, J. Multi-crop convolutional neural networks for lung nodule malignancy suspiciousness classification. Pattern Recognition 61 (2017), 663-673.

[34] Singh, D., Kumar, V., Kaur, M., Et Al. Classification of covid-19 patients from chest ct images using multi-objective differential evolution-based convolutional neural networks. European Journal of Clinical Microbiology 63 Infectious Diseases 39, 7 (2020), 1379-1389.

[35] Song, Q., Zhao, L., Luo, X., And Dou, X. Using deep learning for classification of lung nodules on computed tomography images. Journal of healthcare engineering 2017 (2017).

[36] Stephen, O., Sain, M., Maduh, U. J., And JEONG, D.-U. An efficient deep learning approach to pneumonia classification in healthcare. Journal of healthcare engineering 2019 (2019).

[37] ToĞAÇAr, M., Ergen, B., And Cömert, Z. Covid-19 detection using deep learning models to exploit social mimic optimization and structured chest x-ray images using fuzzy color and stacking approaches. Computers in biology and medicine 121 (2020), 103805.

[38] Vangipuram, S. K., And Appusamy, R. Machine learning framework for covid-19 diagnosis. In International Conference on Data Science, Elearning and Information Systems 2021 (2021), pp. $18-25$.

[39] Vangipuram, S. K., And Appusamy, R. A survey on similarity measures and machine learning algorithms for classification and prediction. In International Conference on Data Science, Elearning and Information Systems 2021 (2021), pp. 198-204.

[40] Waheed, A., Goyal, M., Gupta, D., Khanna, A., Al-Turjman, F., And Pinheiro, 
P. R. Covidgan: data augmentation using auxiliary classifier gan for improved covid-19 detection. Ieee Access 8 (2020), 91916-91923.

[41] Wang, C., Elazab, A., Wu, J., and Hu, Q. Lung nodule classification using deep feature fusion in chest radiography. Computerized Medical Imaging and Graphics 57 (2017), 10-18.

[42] Wang, L., Lin, Z. Q., And Wong, A. Covidnet: A tailored deep convolutional neural network design for detection of covid-19 cases from chest x-ray images. Scientific Reports 10, 1 (2020), 112.

[43] Wang, S., Kang, B., Ma, J., Zeng, X., XiaO, M., Guo, J., Cai, M., Yang, J., Li, Y., Meng, $\mathrm{X}$., ET AL. A deep learning algorithm using ct images to screen for corona virus disease (covid19). European radiology (2021), 1-9.

[44] Wang, S., Zha, Y., LI, W., Wu, Q., LI, X., Niu, M., Wang, M., QIU, X., Li, H., Yu, H., ET AL. A fully automatic deep learning system for covid-19 diagnostic and prognostic analysis. European Respiratory Journal 56, 2 (2020).

[45] Wang, X., Deng, X., Fu, Q., Zhou, Q., Feng, J., Ma, H., Liu, W., And Zheng, C. A weaklysupervised framework for covid-19 classification and lesion localization from chest ct. IEEE transactions on medical imaging 39, 8 (2020), 26152625.

[46] Xie, H., Yang, D., Sun, N., Chen, Z., And ZHANG, Y. Automated pulmonary nodule detection in ct images using deep convolutional neural networks. Pattern Recognition 85 (2019), 109119.

[47] Xu, X., Jiang, X., Ma, C., Du, P., LI, X., Lv, S., Yu, L., Ni, Q., Chen, Y., Su, J., Et Al. A deep learning system to screen novel coronavirus disease 2019 pneumonia. Engineering 6, 10 (2020), $1122-1129$.

[48] Zhang, K., Liu, X., Shen, J., Li, Z., Sang, Y., Wu, X., Zha, Y., Liang, W., Wang, C., WANG, K., ET AL. Clinically applicable ai system for accurate diagnosis, quantitative measurements, and prognosis of covid-19 pneumonia using computed tomography. Cell 181, 6 (2020), 14231433. 